\title{
Valor predictivo de la historia clínica y examen físico en el diagnóstico de neumonía del adulto adquirida en la comunidad
}

\author{
Fernando Saldías P1,2, D aniel Cabrera $\mathrm{T}^{2}$, \\ Ignacio de Solminihac $L^{a}$, Pamela Hernández $A^{a}$, \\ Alessandra Gederlini $\mathbf{G}^{3}$, Alejandro Díaz $\mathrm{F}^{1}$. \\ Predictive value of history and \\ physical examination for the \\ diagnosis of community-acquired \\ pneumonia in adults
}

Background: Community-acquired pneumonia in adults is a serious health problem in the ambulatory care setting. Aim: To define clinical variables associated with the presence of pneumonia in adult patients presenting with fever or respiratory symptoms to the emergency department. Material and methods: Prospective study carried out in the emergency department from the Catholic University Hospital in Santiago, Chile. Three hundred twenty-five patients (53 \pm 22 years) presenting fever or acute respiratory symptoms were included. After obtaining a clinical history and physical examination, the physician established a tentative diagnosis. Subsequently, a definitive diagnosis was made with the chest $\mathrm{X}$ rays. Results: Thirty-four percent of the patients had pneumonia. The clinical diagnosis of pneumonia before X-ray examination was variable among emergency physicians (positive likelihood ratio: 1.5-4.8) and showed only moderate sensitivity (79\%) and specificity (66\%). The clinical variables significantly associated with the presence of pneumonia were: advanced age (over 75 years), cardiovascular disease, fever, chills, sputum production, orthopnea, altered mental status, cyanosis, dullness on percussion, bronchial breath sounds, crackles, any abnormal vital sign (heart rate $\geq 100$ beats/min, respiratory rate $\geq 20$ breaths/min or temperature $\geq 38^{\circ} \mathrm{C}$ ) and oxygen saturation below $90 \%$ breathing air. Conclusions: Clinical judgment prior to observation of chest $\mathrm{X}$ rays had moderate sensitivity and specificity for the diagnosis of pneumonia. There were no individual clinical findings, or combination of findings, that could confirm or exclude the diagnosis of pneumonia for a patient suspected of having this illness (Rev Méd Chile 2007; 135: 143-52).

(Key words: Diagnosis, differential; Pneumonia; Radiography, thoracic)

Recibido el 17 de mayo, 2006. Aceptado el 22 de agosto, 2006.

${ }^{1}$ Departamento de Enfermedades Respiratorias, ${ }^{2}$ Medicina de Urgencia y ${ }^{3}$ Bioestadística de la Pontificia Universidad Católica de Chile.

aAlumno de Medicina, Pontificia Universidad Católica de Chile.

Correspondencia a: Dr. Fernando Saldías P. Departamento de Enfermedades Respiratorias, Pontificia Universidad Católica de Chile. Marcoleta 352. Santiago, Chile. Teléfonos: (562)6331541(562)3543242. Fax: (562)6335255. E mail: fsaldias@med.puc.cl 
T a neumonía adquirida en la comunidad (NAC) Lconstituye una causa frecuente de consulta ambulatoria, hospitalización y muerte en la población adulta de los países desarrollados y en vías de desarrollo ${ }^{1-3}$. Se ha estimado que cerca de $4 \%$ $5 \%$ de las consultas en los servicios de atención primaria (consultorios y servicios de urgencia) son por síntomas respiratorios agudos, correspondiendo a la neumonía el quinto lugar con $5 \%$ del total de visitas por patologías respiratorias, después de la bronquitis aguda, infecciones del tracto respiratorio superior, asma bronquial y sinusitis ${ }^{4}$. Aunque la neumonía puede representar sólo una pequeña proporción de las enfermedades respiratorias agudas que consultan en los servicios de atención primaria, la adecuada pesquisa de este subgrupo es muy importante, considerando su particular riesgo de complicaciones y muerte. Además, el tratamiento antibiótico empírico es considerado universalmente, en las distintas guías clínicas, como la principal herramienta terapéutica en el manejo del paciente con neumonía comunitaria ${ }^{5-7}$.

En el ámbito ambulatorio, el diagnóstico de la neumonía comunitaria se sustenta en un cuadro respiratorio agudo, caracterizado por compromiso del estado general, fiebre, calofríos, tos, expectoración purulenta o dificultad respiratoria; asociado en el examen físico a taquicardia, taquipnea, temperatura corporal elevada, y signos focales en el examen pulmonar ${ }^{8}$. Sin embargo, esta descripción no ha sido planteada en términos de probabilidad, que suele ser la realidad de la práctica clínica, en la que debemos definir si iniciamos tratamiento antibiótico con los elementos clínicos entregados por la historia y examen físico, o debemos solicitar exámenes de laboratorio complementarios. Esta decisión no es trivial, ya que la neumonía es la única infección respiratoria aguda en que un retardo en el inicio del tratamiento antibiótico se ha asociado a un mayor riesgo de complicaciones y muerte ${ }^{9-11}$.

La radiografía de tórax permite confirmar el diagnóstico clínico de neumonía, establecer su localización, extensión y gravedad, y puede ser útil en el seguimiento de los pacientes de alto riesgo ${ }^{12,13}$. Es posible que algunos médicos de atención primaria aún establezcan el diagnóstico de neumonía basados exclusivamente en elementos clínicos, mientras que otros soliciten radiografía de tórax para confirmar el diagnóstico clínico en todos los enfermos. En la práctica ambulatoria, se desconoce la proporción de médicos que siguen una u otra estrategia diagnóstica.

La probabilidad de un paciente con síntomas respiratorios agudos de tener una neumonía depende de la prevalencia de la enfermedad en el ambiente donde se presenta y de las manifestaciones clínicas del enfermo ${ }^{14-18}$. Es por ello que diversos estudios han comunicado tasas de prevalencia de neumonía muy variables, oscilando entre 2,6\% y 38\%. En general, la prevalencia de esta enfermedad aumenta en la población senescente con comorbilidad múltiple y pobre acceso a los servicios de salud, y disminuye en el adulto joven, sin comorbilidad y con buen acceso a la atención medica ${ }^{1-3}$

En la última década, la Sociedad Americana de Tórax (ATS) y la Sociedad Británica de Tórax (BTS), han actualizado sus guías clínicas sobre el manejo de la neumonía comunitaria del adulto inmunocompetente ${ }^{5,6}$. La Sociedad Americana de Tórax recomienda solicitar radiografía de tórax a todos los pacientes con sospecha clínica de neumonía; mientras que la Sociedad Británica de Tórax no recomienda el uso rutinario de radiografía de tórax en el manejo ambulatorio de pacientes con neumonía, considerando las características del sistema de salud del Reino Unido. El propósito de este estudio ha sido describir las variables clínicas de la historia y examen físico que permiten predecir el diagnóstico de neumonía en adultos inmunocompetentes atendidos por fiebre y síntomas respiratorios en la unidad de emergencia de un hospital docente de Santiago.

\section{PACIENTES Y MÉTODO}

Se evaluaron en forma prospectiva 325 pacientes adultos atendidos por fiebre o síntomas respiratorios agudos (tos, expectoración, disnea o dolor torácico) en la Unidad de Emergencia del Hospital Clínico de la Pontificia Universidad Católica de Chile (hospital docente de 520 camas) entre el $1^{0}$ de enero y 31 de diciembre de 2005. Se incluyeron en el estudio los pacientes inmunocompetentes mayores de 15 años que cumplieron los criterios clínicos descritos y fueron evaluados por tres médicos del Servicio de Urgencia que participaron en el protocolo (FS, DC, $\mathrm{AD}$ ), quienes 
solicitaron una radiografía de tórax para la evaluación de sus síntomas. Se excluyeron del estudio los pacientes con daño pulmonar crónico e infiltrados radiográficos persistentes que dificultarían la interpretación de la radiografía de tórax (bronquiectasias, fibrosis pulmonar, secuelas de tuberculosis), infección por virus de inmunodeficiencia humana (VIH), tumores sólidos o hematológicos, neutropenia (recuento de leucocitos menor de $1.000 / \mathrm{mm}^{3}$ ), tratamiento quimioterápico o inmunosupresor en los seis meses previos a la consulta o el antecedente de neumonía u hospitalización en los últimos tres meses.

En los pacientes ingresados al protocolo, los médicos de urgencia registraron los siguientes antecedentes clínico-epidemiológicos: edad, sexo, motivo de consulta, duración de los síntomas, presencia de comorbilidad, consumo de tabaco, uso de antibióticos previo a la consulta (cualquier dosis recibida de un antimicrobiano en la última semana), cuadro clínico de presentación, hallazgos del examen físico pulmonar, signos vitales y saturación arterial de oxígeno medida por oxímetro de pulso. Una vez evaluado el paciente, el médico consignaba su hipótesis diagnóstica y la probabilidad de neumonía antes de recibir el informe de la radiografía de tórax.

A todos los pacientes se les realizó una radiografía de tórax posteroanterior y lateral en el Servicio de Radiología, la cual fue evaluada por un radiólogo del Departamento de Radiología involucrado en el estudio, quien desconocía el cuadro clínico de los pacientes. El patrón radiográfico correspondió a alguna de las siguientes categorías: a) normal; b) imagen de relleno alveolar, c) patrón intersticial, d) patrón mixto, e) otros hallazgos radiográficos tales como cardiomegalia, congestión pulmonar, derrame pleural, neumotórax, etc. El diagnóstico definitivo fue establecido por el médico de urgencia en los pacientes ambulatorios con radiografía de tórax normal, y por los investigadores (IS, PH), en los pacientes con infiltrados radiográficos que fueron admitidos al hospital o referidos al consultorio externo de medicina, donde se consignaron los diagnósticos establecidos en el seguimiento a 30 días. El diagnóstico de neumonía fue establecido por la demostración de un infiltrado pulmonar de aparición reciente en la radiografía de tórax que se resolvió con tratamiento antibiótico y no corres- pondía a un diagnóstico alternativo en el seguimiento a 30 días.

Estadística. Los resultados fueron expresados como valores promedio \pm desviación estándar para las variables medidas en escala numérica y en porcentaje para las medidas en escala nominal. Las variables continuas fueron manejadas como variables dicotómicas, considerando su mejor punto de corte de sensibilidad y especificidad en las curvas ROC (receiver operating characteristic curve). La asociación entre las variables clínicas (síntomas y signos) y los hallazgos radiográficos (presencia 0 ausencia de neumonía) fue determinada mediante la prueba de chi cuadrado o test exacto de Fisher. Se midió la sensibilidad (S), especificidad (E), valor predictivo positivo (VPP) y negativo (VPN) y la razón de verosimilitud o likelihood ratio positivo $(\mathrm{L}+)$ de las variables clínicas que fueron significativas en el análisis bivariado con un valor de $\mathrm{p}$ $<0,05$. Las variables clínicas que resultaron significativas fueron incorporadas a un modelo de regresión logística (modalidad stepwise) que permite el control simultáneo de múltiples factores. De este modo, los parámetros que no agregaron valor predictivo no fueron retenidos en el modelo. Se calcularon los odds ratio e intervalos de confianza (IC) para $95 \%$.

\section{RESULTADOS}

Durante el período del estudio, se evaluaron 325 adultos inmunocompetentes con fiebre o síntomas respiratorios agudos en la Unidad de Emergencia. Las características clínicas de los pacientes son descritas en la Tabla 1. La edad promedio fue $53 \pm 22$ años (rango: 15-100), 59\% eran mujeres, 51\% tenía alguna comorbilidad (especialmente cardiovascular y respiratoria crónica), 31\% eran fumadores, la duración promedio de los síntomas que motivaron la consulta fue siete días y en un tercio de los casos se estableció el diagnóstico de neumonía comunitaria. Los principales diagnósticos alternativos fueron bronquitis aguda (34\%), gripe 0 influenza (13\%), exacerbación de enfermedad pulmonar obstructiva crónica o asma bronquial (6\%), e infecciones del tracto respiratorio superior (4\%).

En la Tabla 2 se describe los principales hallazgos de la historia clínica y el examen físico 
Tabla 1. Características clínicas de los pacientes adultos atendidos por fiebre o síntomas respiratorios agudos en el servicio de urgencia

\begin{tabular}{|lcc|}
\hline Variables & $\mathrm{n}$ & $\%$ \\
\hline $\mathrm{N}$ & 325 & \\
Edad (años) $(\mathrm{x} \pm \mathrm{ds})$ & $53,4 \pm 22,6$ & $(\mathrm{R}: 15-100)$ \\
Sexo $(\mathrm{M} / \mathrm{F})(\mathrm{n}, \%)$ & $132 / 193$ & 31 \\
Fumadores & 100 & 51 \\
Comorbilidad & 167 & 17 \\
Enfermedad cardiovascular & 54 & 13 \\
EPOC-Asma bronquial & 41 & 10 \\
Diabetes mellitus & 32 & 4 \\
Enfermedad neurológica crónica & 14 & 2 \\
Insuficiencia renal crónica & 6 & $(\mathrm{R}: 1-30)$ \\
Duración de los síntomas (días) & $7,1 \pm 6,3$ & 34,5 \\
Diagnósticos definitivos: & & 33,5 \\
$\quad$ Neumonía comunitaria & 112 & 12,6 \\
Bronquitis aguda & 109 & 3,7 \\
Gripe-Influenza & 41 & 3,4 \\
Infección tracto respiratorio superior & 12 & 2,8 \\
EPOC descompensado & 11 & 2,8 \\
Crisis asmática & 9 & \\
Síndrome febril & 9 & \\
\hline
\end{tabular}

Nota: M: masculino, F: femenino, EPOC: enfermedad pulmonar obstructiva crónica.

que se asociaron con el diagnóstico de neumonía comunitaria: edad avanzada ( $>75$ años), enfermedad cardiovascular, fiebre, escalofríos, producción de esputo, ortopnea, confusión mental, cianosis, tiraje, matidez torácica, broncofonia, crepitaciones, alteración de los signos vitales (taquicardia $\geq 100$ lat/min, taquipnea $\geq 20 \mathrm{resp} / \mathrm{min}$ o temperatura $\geq 38^{\circ} \mathrm{C}$ ) y saturación arterial de oxígeno bajo $90 \%$ respirando aire ambiente. La presencia de las siguientes comorbilidades, enfermedad pulmonar obstructiva crónica, asma bronquial, diabetes mellitus y enfermedad neurológica crónica, fue similar en los pacientes con neumonía u otro diagnóstico alternativo. No hubo relación entre la duración de los síntomas, la estación del año (otoño-invierno versus primavera-verano), la presencia de tos, disnea, dolor torácico, mialgias o sudoración nocturna y el diagnóstico de neumonía.

La sensibilidad y especificidad del juicio clínico de los tres médicos de urgencia, antes de ver la radiografía de tórax, para establecer el diagnóstico de neumonía oscilaron entre $75-83 \%$ y $47-83 \%$, respectivamente (Tabla 3). El diagnóstico clínico de neumonía en la unidad de emergencia tuvo una sensibilidad de $79 \%$, especificidad de $66 \%$, valor predictivo positivo de $55 \%$ y valor predictivo negativo de $85 \%$. La probabilidad de modificar el diagnóstico inicial de neumonía o la razón de verosimilitud (LR+) del juicio clínico de los tres médicos de urgencia varió entre 1,5 y 4,8.

En la Tabla 4 se exponen la sensibilidad, especificidad, valor predictivo positivo y negativo de los principales hallazgos clínicos (historia y examen físico) asociados al diagnóstico de neumonía en el servicio de urgencia. Los principales predictores clínicos de neumonía fueron la fiebre $\left(\geq 38^{\circ} \mathrm{C}\right)$, taquipnea ( $\left.\geq 30 \mathrm{resp} / \mathrm{min}\right)$, estado mental alterado, ortopnea, cianosis, tiraje, matidez torácica, broncofonia, crepitaciones y $\mathrm{SaO}_{2}<90 \%$ respirando aire ambiente $\left(\mathrm{LR}^{+}\right.$entre $\left.2,0-9,5\right)$. La odinofagia, rinorrea, signos vitales y auscultación pulmonar normales fueron menos frecuentes en los pacientes con diagnóstico definitivo de neumonía $\left(\mathrm{LR}^{+}\right.$entre $\left.0,3-0,5\right)$. En la Tabla 5, se describe algunas combinaciones de variables clínicas que incrementan la probabilidad de neumo- 
Tabla 2. Variables clínicas asociadas a la presencia de neumonía en adultos que consultan por fiebre o síntomas respiratorios agudos en el servicio de urgencia

\begin{tabular}{|lccc|}
\hline Variables clínicas & Neumonía & Otros diagnósticos & $\mathrm{p}$ \\
\hline $\mathrm{N}$ & 112 & 213 & \\
Edad $>75$ años & $32(29 \%)$ & $36(17 \%)$ & 0,02 \\
Cardiopatía & $25(22 \%)$ & $29(14 \%)$ & 0,05 \\
Fiebre & $90(80 \%)$ & $131(62 \%)$ & 0,001 \\
Escalofríos & $60(54 \%)$ & $79(37 \%)$ & 0,005 \\
Tos & $103(92 \%)$ & $194(91 \%)$ & 0,78 \\
Expectoración & $81(72 \%)$ & $130(61 \%)$ & 0,05 \\
Odinofagia & $20(18 \%)$ & $75(35 \%)$ & 0,001 \\
Rinorrea & $25(22 \%)$ & $85(40 \%)$ & 0,001 \\
Ortopnea & $21(19 \%)$ & $16(8 \%)$ & 0,003 \\
Confusión mental & $10(9 \%)$ & $8(4 \%)$ & 0,05 \\
Cianosis & $17(15 \%)$ & $7(3 \%)$ & 0,001 \\
Tiraje & $11(10 \%)$ & $4(2 \%)$ & 0,002 \\
Matidez & $18(16 \%)$ & $9(4 \%)$ & 0,001 \\
Broncofonia & $5(4 \%)$ & $1(0,5 \%)$ & 0,02 \\
Crepitaciones & $66(59 \%)$ & $63(30 \%)$ & 0,001 \\
Auscultación pulmonar anormal & $95(85 \%)$ & $139(65 \%)$ & 0,001 \\
F. cardíaca $\geq 100$ lat/min & $55(49 \%)$ & $77(36 \%)$ & 0,03 \\
F. respiratoria $\geq 20$ resp/min & $96 / 107(90 \%)$ & $136 / 196(69 \%)$ & 0,001 \\
Fiebre $\geq 38^{\circ} \mathrm{C}$ & $40 / 108(37 \%)$ & $35 / 207(17 \%)$ & 0,001 \\
SaO $<$ <0\% (FiO $:$ 0,21) & $30 / 110(27 \%)$ & $13 / 201(6 \%)$ & 0,001 \\
Signos vitales anormales & $89 / 103(86 \%)$ & $108 / 193(56 \%)$ & 0,001 \\
& & & \\
\hline
\end{tabular}

Nota: El número del denominador corresponde a los pacientes con la variable registrada.

Auscultación pulmonar anormal: presencia de broncofonia, roncus, crepitaciones o sibilancias en el examen físico pulmonar. Signos vitales anormales: frecuencia cardíaca $\geq 100 \mathrm{lat} / \mathrm{min}$, frecuencia respiratoria $\geq 20 \mathrm{resp} /$ min o temperatura axilar $\geq 37,8^{\circ} \mathrm{C}$.

Tabla 3. Sensibilidad, especificidad, valor predictivo positivo (VPP) y negativo (VPN) y razón de verosimilitud $\left(\mathrm{LR}^{+}\right)$del juicio clínico para la pesquisa de neumonía en la unidad de emergencia

\begin{tabular}{|lccccc|}
\hline Juicio clínico & Sensibilidad & Especificidad & VPP & VPN & LR $^{+}$ \\
\hline Médico A & $83 \%$ & $83 \%$ & $70 \%$ & $91 \%$ & 4,8 \\
Médico B & $75 \%$ & $73 \%$ & $56 \%$ & $86 \%$ & 2,8 \\
Médico C & $77 \%$ & $47 \%$ & $36 \%$ & $85 \%$ & 1,5 \\
Promedio & $79 \%$ & $66 \%$ & $55 \%$ & $85 \%$ & 2,3 \\
\hline
\end{tabular}

Nota: Médicos A y B: especialistas en medicina interna y enfermedades respiratorias con más de 5 años de ejercicio profesional, Médico C: especialista en medicina de ungencia con menos de 3 años de ejercicio profesional. 
Tabla 4. Sensibilidad, especificidad, valor predictivo positivo y negativo y razón de verosimilitud de las variables clínicas para el diagnóstico de neumonía en el servicio de urgencia

\begin{tabular}{|c|c|c|c|c|c|}
\hline Variables clínicas & Sensibilidad & Especificidad & VPP & VPN & $\mathrm{LR}^{+}$ \\
\hline Edad $>75$ años & $29 \%$ & $83 \%$ & $47 \%$ & $69 \%$ & 1,7 \\
\hline Cardiopatía & $22 \%$ & $86 \%$ & $46 \%$ & $68 \%$ & 1,6 \\
\hline Fiebre & $80 \%$ & $39 \%$ & $41 \%$ & $79 \%$ & 1,3 \\
\hline Escalofríos & $54 \%$ & $63 \%$ & $43 \%$ & $72 \%$ & 1,4 \\
\hline Tos & $92 \%$ & $9 \%$ & $35 \%$ & $68 \%$ & 1,0 \\
\hline Expectoración & $72 \%$ & $39 \%$ & $38 \%$ & $73 \%$ & 1,2 \\
\hline Odinofagia & $18 \%$ & $65 \%$ & $21 \%$ & $60 \%$ & 0,5 \\
\hline Rinorrea & $22 \%$ & $60 \%$ & $23 \%$ & $60 \%$ & 0,5 \\
\hline Ortopnea & $19 \%$ & $92 \%$ & $57 \%$ & $68 \%$ & 2,5 \\
\hline Confusión mental & $9 \%$ & $96 \%$ & $56 \%$ & $67 \%$ & 2,4 \\
\hline Cianosis & $15 \%$ & $97 \%$ & $71 \%$ & $68 \%$ & 4,6 \\
\hline Tiraje & $10 \%$ & $98 \%$ & $73 \%$ & $67 \%$ & 5,2 \\
\hline Matidez & $16 \%$ & $96 \%$ & $67 \%$ & $68 \%$ & 3,8 \\
\hline Broncofonia & $4 \%$ & $99 \%$ & $83 \%$ & $66 \%$ & 9,5 \\
\hline Crepitaciones & $59 \%$ & $70 \%$ & $51 \%$ & $77 \%$ & 2,0 \\
\hline Auscultación pulmonar normal & $15 \%$ & $65 \%$ & $19 \%$ & $59 \%$ & 0,4 \\
\hline F. cardíaca $\geq 100 \mathrm{x}^{\prime}$ & $49 \%$ & $64 \%$ & $42 \%$ & $70 \%$ & 1,4 \\
\hline F. respiratoria $\geq 20 \mathrm{x}^{\prime}$ & $90 \%$ & $31 \%$ & $41 \%$ & $85 \%$ & 1,3 \\
\hline F. respiratoria $\geq 30 \mathrm{x}^{\prime}$ & $32 \%$ & $85 \%$ & $53 \%$ & $69 \%$ & 2,1 \\
\hline $\mathrm{T} \geq 38^{\circ} \mathrm{C}$ & $37 \%$ & $83 \%$ & $53 \%$ & $72 \%$ & 2,2 \\
\hline $\mathrm{T} \geq 39^{\circ} \mathrm{C}$ & $14 \%$ & $94 \%$ & $56 \%$ & $68 \%$ & 2,4 \\
\hline $\mathrm{SaO}_{2}<90 \%$ & $27 \%$ & $94 \%$ & $70 \%$ & $70 \%$ & 4,2 \\
\hline Signos vitales anormales & $86 \%$ & $44 \%$ & $45 \%$ & $86 \%$ & 1,5 \\
\hline
\end{tabular}

Nota: VPP: Valor predictivo positivo, VPN: Valor predictivo negativo, LR: Likelihood ratio, T: Temperatura axilar, $\mathrm{SaO}_{2}$ : Saturación arterial de oxígeno. Signos vitales anormales: frecuencia cardiaca $\geq 100 \mathrm{lat} / \mathrm{min}$, frecuencia respiratoria $\geq 20 \mathrm{resp} / \mathrm{min} \mathrm{y} / \mathrm{o}$ temperatura axilar $\geq 37,8^{\circ} \mathrm{C}$.

Tabla 5. Sensibilidad, especificidad, valor predictivo positivo y negativo y razón de verosimilitud de combinaciones de variables clínicas para el diagnóstico de neumonía en el servicio de urgencia.

\begin{tabular}{|lcccccc|}
\hline Variables clínicas & Sensibilidad & Especificidad & VPP & VPN & LR $^{+}$ & $\mathrm{p}$ \\
\hline Fiebre + Expectoración & $59 \%$ & $64 \%$ & $47 \%$ & $75 \%$ & 1,7 & 0,001 \\
Fiebre + Escalofríos & $51 \%$ & $66 \%$ & $44 \%$ & $72 \%$ & 1,5 & 0,003 \\
Fiebre + FR $\geq 30 \mathrm{x}^{\prime}$ & $24 \%$ & $92 \%$ & $63 \%$ & $69 \%$ & 3,2 & 0,001 \\
Fiebre + Escalofríos + FR $\geq 30 \mathrm{x}^{\prime}$ & $13 \%$ & $95 \%$ & $58 \%$ & $67 \%$ & 2,6 & 0,02 \\
Fiebre + Ortopnea + FR $\geq 30 \mathrm{x}^{\prime}$ & $7 \%$ & $99 \%$ & $89 \%$ & $66 \%$ & 14,7 & 0,001 \\
$\mathrm{FR} \geq 20 \mathrm{x}^{\prime}+\mathrm{T} \geq 38^{\circ} \mathrm{C}$ & $32 \%$ & $88 \%$ & $58 \%$ & $71 \%$ & 2,6 & 0,001 \\
$\mathrm{FR} \geq 20 \mathrm{x}^{\prime}+\mathrm{T} \geq 38^{\circ} \mathrm{C}+$ Matidez & $4 \%$ & $99 \%$ & $80 \%$ & $66 \%$ & 7,5 & 0,04 \\
$\mathrm{FR} \geq 20 \mathrm{x}^{\prime}+\mathrm{T} \geq 38^{\circ} \mathrm{C}+\mathrm{Crepitaciones}$ & $20 \%$ & $96 \%$ & $72 \%$ & $69 \%$ & 4,9 & 0,001 \\
$\mathrm{FR} \geq 20 \mathrm{x}^{\prime}+\mathrm{T} \geq 38^{\circ} \mathrm{C}+\mathrm{SaO}_{2}<90 \%$ & $8 \%$ & $99 \%$ & $89 \%$ & $66 \%$ & 14,6 & 0,001 \\
Signos vitales normales & $14 \%$ & $56 \%$ & $14 \%$ & $55 \%$ & 0,3 & 0,001 \\
Signos vitales + examen pulmonar normal & $2 \%$ & $81 \%$ & $5 \%$ & $61 \%$ & 0,1 & 0,001 \\
Odinofagia + Rinorrea & $9 \%$ & $74 \%$ & $15 \%$ & $61 \%$ & 0,3 & 0,001 \\
Odinofagia + Rinorrea + examen pulmonar normal & $1 \%$ & $88 \%$ & $4 \%$ & $63 \%$ & 0,1 & 0,001 \\
\hline
\end{tabular}

Nota: VPP: Valor predictivo positivo, VPN: Valor predictivo negativo, LR: Likelihood ratio, FR: Frecuencia respiratoria, T: Temperatura axilar, $\mathrm{SaO}_{2}$ : Saturación arterial de oxígeno.

Signos vitales normales: frecuencia cardiaca $<100$ lat/min, frecuencia respiratoria $<20 \mathrm{resp} / \mathrm{min}$ y temperatura axilar $<37,8^{\circ} \mathrm{C}$. 
nía, tales como fiebre, taquipnea o ambas asociados a ortopnea, matidez torácica, crepitaciones o $\mathrm{SaO}_{2}<90 \%$ (LR+ entre 4,9-14,7). La probabilidad de neumonía es muy baja en el paciente que consulta por síntomas respiratorios y tiene signos vitales y examen pulmonar normales $\left(\mathrm{LR}^{+}: 0,1\right)$.

En el análisis de regresión logística multivariado, las principales variables clínicas asociadas con el diagnóstico de neumonía fueron la fiebre $\geq 38^{\circ} \mathrm{C}$, taquipnea $\geq 20 \mathrm{resp} / \mathrm{min}, \mathrm{SaO}_{2}<90 \%$, y la presencia de matidez torácica y crepitaciones en el examen pulmonar (Tabla 6).

\section{DisCUSIÓN}

El principal objetivo del estudio fue describir las variables clínicas que se asocian al diagnóstico de neumonía en el adulto inmunocompetente que consulta por síntomas respiratorios, fiebre, 0 ambos en la unidad de emergencia. Los principales hallazgos del estudio fueron: 1) El diagnóstico clínico de neumonía realizado por los médicos del servicio de urgencia tuvo una sensibilidad y especificidad variable y de magnitud moderada; 2) Los elementos aislados de la historia y examen físico no permitieron confirmar ni descartar con seguridad el diagnóstico de neumonía sin solicitar una radiografía de tórax; 3) La combinación de variables clínicas permitió incrementar o reducir la probabilidad de neumonía comunitaria comparado con las variables aisladas; 4) Los signos vitales y la auscultación pulmonar normales redujeron en forma significativa la probabilidad de neumonía;
5) La edad, comorbilidad, hábito tabáquico, duración de los síntomas y la estacionalidad no se asociaron de forma significativa con el diagnóstico de neumonía.

Los síntomas respiratorios, especialmente la tos, son motivo frecuente de consulta en los servicios de atención primaria, representando entre $4 \%-5 \%$ de las consultas 4 . Tradicionalmente, el paciente es evaluado mediante una historia clínica y examen físico, y suele solicitarse una radiografía de tórax (en uno de cada 7-8 casos) y otros exámenes complementarios según el riesgo individual. La principal razón que tiene el clínico para solicitar una radiografía de tórax, es para confirmar o descartar el diagnóstico de neumonía, considerando su pronóstico particular y tratamiento específico ${ }^{5-8}$. Los criterios utilizados por los clínicos para obtener una radiografía de tórax en adultos que consultan por síntomas respiratorios aún no han sido claramente establecidos. Este estudio fue diseñado para examinar el valor predictivo de los hallazgos clínicos (síntomas y signos), en pacientes que consultan por fiebre 0 síntomas respiratorios agudos, en la pesquisa de episodios de neumonía confirmados por radiología y seguimiento clínico.

El diagnóstico clínico de neumonía realizado por los médicos en la unidad de emergencia tuvo mejor sensibilidad (rango: 75-83\%) que especificidad (rango: 47-83\%), y mejor valor predictivo negativo (rango: 85-91\%) que positivo (rango: 3670\%). En el diagnóstico de neumonía, el médico de urgencia menos experimentado (médico $\mathrm{C}$ ) tuvo menor valor predictivo positivo y especificidad

\section{Tabla 6. Variables clínicas asociadas al diagnóstico de neumonía en adultos que consultan por fiebre o síntomas respiratorios agudos en el servicio de urgencia. Análisis multivariado}

\begin{tabular}{|lcccc|}
\hline Variables clínicas & $\begin{array}{c}\text { Pacientes } \\
(\mathrm{n})\end{array}$ & $\begin{array}{c}\text { Neumonía } \\
(\mathrm{n})\end{array}$ & Odds ratio & IC 95\% \\
\hline Temperatura $\geq 38^{\circ} \mathrm{C}$ & 75 & 40 & 3,4 & $1,8-6,4$ \\
$\mathrm{SaO}_{2}<90 \%$ & 43 & 30 & 3,2 & $1,5-7,2$ \\
$\mathrm{Matidez}$ torácica & 27 & 18 & 3,1 & $1,1-8,8$ \\
Crepitaciones & 129 & 66 & 2,8 & $1,6-4,8$ \\
F. respiratoria $\geq 20 \mathrm{x}^{\prime}$ & 232 & 96 & 2,4 & $1,1-5,1$ \\
\hline
\end{tabular}

Nota: IC 95\%: intervalo de confianza de 95\%, $\mathrm{SaO}_{2}$ : saturación arterial de oxígeno. 
comparado con los médicos especialistas en medicina interna y enfermedades respiratorias (médicos A y B). Hallazgos similares fueron descritos por Wipf y cols, quienes evaluaron la precisión y variabilidad de los examinadores en la pesquisa de los signos clínicos en pacientes con y sin diagnóstico de neumonía ${ }^{19}$. Los autores concluyeron que los hallazgos semiológicos pesquisados en el examen de tórax no permiten confirmar ni excluir con seguridad el diagnóstico de neumonía, existiendo gran variabilidad interobservador en la pesquisa de hallazgos semiológicos específicos. González y cols, en un estudio prospectivo realizado en dos servicios de urgencia de Madrid, señalaron que el juicio clínico tiene una baja sensibilidad para el diagnóstico de neumonía (45\%) con un moderado valor predictivo positivo $(80 \%)^{20}$.

Las principales variables clínicas que se asociaron con la presencia de neumonía fueron la edad avanzada, comorbilidad cardiovascular, fiebre, es- calofríos, producción de esputo, ortopnea, confusión mental, cianosis, tiraje, matidez torácica, broncofonia, crepitaciones, alteración de los signos vitales y $\mathrm{SaO}_{2}$ menor de $90 \%$. Similar a lo descrito en la literatura (Tabla 7), en el análisis multivariado permanecieron asociadas con el diagnóstico de neumonía las siguientes variables: fiebre $\geq 38^{\circ} \mathrm{C}$, taquipnea $\geq 20 \mathrm{resp} / \mathrm{min}, \mathrm{SaO}_{2}<90 \%$, matidez y crepitaciones en el examen pulmonar. Sin embargo, los elementos aislados de la historia y examen físico no permitieron confirmar ni descartar con seguridad el diagnóstico de neumonía, con valores de $\mathrm{LR}^{+}$entre 0,4 y 5,2. Sólo el hallazgo de broncofonia tuvo un $\mathrm{LR}^{+}$cercano a diez, pero fue un hallazgo semiológico de excepción (sensibilidad: 4\%) aunque altamente específico (99\%). Similar a lo descrito por Diehr y cols ${ }^{14}$, la presencia de odinofagia y rinorrea disminuyó el riesgo de neumonía (Tabla 7), lo cual probablemente carece de mayor trascendencia clínica.

Tabla 7. Variables clínicas asociadas al diagnóstico de neumonía adquirida en la comunidad en la unidad de emergencia $\left(L^{+}\right)^{14-17}$

\begin{tabular}{|lccccc|}
\hline Variables & Diehr et al & Gennis et al & Singal et al & Heckerling & Saldías et al \\
\hline Tos & - & NS & 1,8 & NS & NS \\
Expectoración & 1,3 & NS & - & NS & 1,2 \\
Disnea & - & 1,4 & NS & NS & NS \\
Fiebre & 2,1 & NS & - & 1,7 & 1,3 \\
Escalofríos & 1,6 & 1,3 & - & 1,7 & 1,4 \\
Mialgias & 1,3 & NS & - & - & NS \\
Odinofagia & 0,7 & NS & - & - & 0,5 \\
Rinorrea & 0,7 & NS & - & - & 0,5 \\
FC $>100 x^{\prime}$ & NS & 1,6 & NS & 2,3 & 1,4 \\
FR $>20 \mathrm{x}^{\prime}$ & - & 1,2 & - & - & 1,3 \\
T >37,8 ${ }^{\circ} \mathrm{C}$ & 4,4 & 1,4 & 2,4 & 2,4 & 2,2 \\
Signos vitales normales & - & 0,2 & - & - & 0,3 \\
Matidez & NS & 2,2 & - & 4,3 & 3,8 \\
Roncus & NS & 1,5 & - & 1,4 & NS \\
Broncofonia & - & - & - & 3,5 & 9,5 \\
Crepitaciones & 2,7 & 1,6 & 1,7 & 2,6 & 2,0 \\
Examen pulmonar normal & - & 0,5 & - & - & 0,4 \\
\hline
\end{tabular}

Nota: $\mathrm{LR}^{+}$: razón de verosimilitud o likelihood ratio positivo. FC: frecuencia cardiaca, FR: frecuencia respiratoria, T: temperatura axilar. Signos vitales normales: frecuencia cardiaca $<100$ lat $/ \mathrm{min}$, frecuencia respiratoria $<20 \mathrm{resp} / \mathrm{min}$ y temperatura axilar $<37,8^{\circ} \mathrm{C}$. NS: variable no significativa. 
La Sociedad Americana de Tórax ha recomendado solicitar radiografía de tórax para confirmar el diagnóstico clínico de neumonía en todos los enfermos; mientras que la Sociedad Británica de Tórax sugiere a los médicos de atención primaria manejar a los pacientes de bajo riesgo exclusivamente con los elementos clínicos de la anamnesis y examen físico, pero recomienda solicitar examen radiográfico a todos los enfermos con criterios de gravedad que son admitidos al hospital ${ }^{5,6}$. Es decir, ambas sociedades científicas reconocen la importancia del examen radiológico para confirmar el diagnóstico, evaluar la gravedad y descartar complicaciones en el seguimiento de los enfermos hospitalizados por neumonía.

La combinación de variables clínicas permitió incrementar o reducir la probabilidad de neumonía comunitaria comparado con la variables aisladas (Tabla 5). De este modo, similar a lo descrito por Gennis y cols ${ }^{15}$, los signos vitales y la

\section{REFERENCIAS}

1. GARIBALd RA. Epidemiology of community-acquired respiratory tract infections in adults: incidence, etiology, and impact. Am J Med 1985; 78: 32-7.

2. Almirall J, Bolbar I, Vidal J, Sauca G, Coll P, NiKLASSON B ET AL. Epidemiology of communityacquired pneumonia in adults: a populationbased study. Eur Respir J 2000; 15: 757-63.

3. GotFRIED MH. Epidemiology of clinically diagnosed community-acquired pneumonia in the primary care setting: results from the 1999-2000 respiratory surveillance program. Am J Med 2001; 111(Suppl 9A): 25S-29S.

4. Metlay JP, StafFord RS, Singer DE. National trends in the use of antibiotics by primary care physicians for adult patients with cough. Arch Intern Med 1998; 158: 1813-8.

5. Niederman MS, Mandell LA, Anzueto A, Bass JB, Broughton WA, CAMpBell GD ET al. Guidelines for the management of adults with communityacquired pneumonia. Diagnosis, assessment of severity, antimicrobial therapy, and prevention. Am J Respir Crit Care Med 2001; 163: 1730-54.

6. British Thoracic Society Standards of Care CommitTEE. British Thoracic Society guidelines for the auscultación pulmonar normales disminuyeron la probabilidad de neumonía en un rango moderado ( $\mathrm{LR}^{+}$entre 0,3 y 0,4) (Tabla 7). Sin embargo, la probabilidad de neumonía es muy baja cuando se presentan ambos hallazgos clínicos, lo cual sólo ocurrió en una baja proporción de enfermos (sensibilidad: 2\%).

En resumen, los hallazgos de la historia clínica y examen físico no permitieron confirmar ni descartar con certeza el diagnóstico de neumonía en adultos inmunocompetentes que consultaron por fiebre o síntomas respiratorios agudos en la unidad de emergencia. Por esta razón, en la guía clínica nacional de manejo de la neumonía comunitaria del adulto ${ }^{7}$, se ha recomendado solicitar examen radiográfico para confirmar el diagnóstico clínico de la infección pulmonar antes de iniciar el tratamiento antibiótico empírico, considerando que el retraso en el diagnóstico se asocia a mayor riesgo de complicaciones y muerte ${ }^{9-11}$.

management of community-acquired pneumonia in adults. Thorax 2001; 56 (suppl IV): 1-64.

7. Sociedad Chilena de Enfermedades Respiratorias y Sociedad Chilena de Infectología. Consenso Nacional 2005: Manejo de la neumonía del adulto adquirida en la comunidad. Rev Chil Enf Respir 2005; 21: 69-140.

8. Metlay JP, Kapoor WN, Fine MJ. Does this patient have community-acquired pneumonia? Diagnosing pneumonia by history and physical examination. JAMA 1997; 278: 1440-5.

9. Meehan TP, Fine MJ, Krumholz HM, Scinto JD, Galusha DH, Mockalis JT et al. Quality of care, process, and outcomes in elderly patients with pneumonia. JAMA 1997; 278: 2080-4.

10. Battleman DS, Caldahan M, Thaler HT. Rapid antibiotic delivery and appropriate antibiotic selection reduce length of hospital stay of patients with community-acquired pneumonia: link between quality of care and resource utilization. Arch Intern Med 2002; 162: 682-8.

11. Houck PM, Bratzier DW, Nsa W, Ma A, Bartlett JG. Timing of antibiotic administration and outcomes for Medicare patients hospitalized with community-acquired pneumonia. Arch Intern Med 2004; 164: 637-44. 
12. WiLKINS TR, WILKINS RL. Clinical and radiographic evidence of pneumonia. Radiol Technol 2005; 77: 106-10.

13. Tarver RD, Teague SD, Heitkamp DE, Conces DJ Jr. Radiology of community-acquired pneumonia. Radiol Clin North Am 2005; 43: 497-512.

14. Diehr P, Wood RW, Bushyhead J, Krueger L, Wolcott B, Tompkins RK. Prediction of pneumonia in outpatients with acute cough - a statistical approach. J Chron Dis 1984; 37: 215-25.

15. Gennis P, Galagher J, Falvo C, Baker S, Than W. Clinical criteria for the detection of pneumonia in adults: guidelines for ordering chest roentgenograms in the emergency department. J Emerg Med 1989; 7: 263-8.

16. Singal BM, Hedges JR, Radack KL Decision rules and clinical prediction of pneumonia: evaluation of low- yield criteria. Ann Emerg Med 1989; 18: 13-20.

17. HeCKERLING PS, TAPE TG, Wigton RS, Hissong KK, LeIKIN JB, ORNato JP ET AL. Clinical prediction rule for pulmonary infiltrates. Ann Intern Med 1990; 113: 664-70.

18. Emerman CL, Dawson N, Speroff T, Siciliano C, EFFRON D, RASHAD F ET AL. Comparison of physician judgment and decision aids for ordering chest radiographs for pneumonia in outpatients. Ann Emerg Med 1991; 20: 1215-19.

19. Wipf JE, Lipsky BA, Hirschmann JV, Boyko EJ, Takasugi J, Peugeot RL et al. Diagnosing pneumonia by physical examination. Relevant or relic? Arch Intern Med 1999; 159: 1082-7.

20. González MA, Carnicero M, Varela M. Predicción de la presencia de neumonía en el adulto con fiebre. Med Clin (Barc) 1995; 105: 521-4.

\section{Agradecimientos}

Los autores agradecen al Dr. Andrés O’Brien S. su valiosa participación en la evaluación de los exámenes radiográficos realizados a los pacientes enrolados en el protocolo. 